\title{
Metodologías interculturales y decoloniales: inclusión de las tradiciones discursivas originarias y rurales en la didáctica
}

\author{
de la lengua \\ Intercultural and Decolonial Methodologies: \\ Inclusion of the Discursive Original and Rural \\ Traditions in the Didactics \\ of Language
}

Fabiola López Ibarra

Dirección de Educación Migrante, México

fabiola.libarra@academicos.udg.mx

\begin{abstract}
RESUMEN
La Dirección de Educación Migrante Jalisco es una institución que depende de la Secretaría de Educación Pública en México. El objetivo del programa es dar atención educativa de calidad a los niños, niñas y adolescentes hijos de familias agrícolas migrantes internas en diversos estados de dicho país. En Jalisco, la atención educativa ha presentado una serie de problemáticas relacionadas con las competencias comunicativas, para ello se ha diseñado una propuesta de intervención pedagógica para lengua oral y escrita en cuatro fases de trabajo. Bajo un enfoque intercultural se fomenta la lengua oral y escrita en contextos donde las culturas orales predominan. El objetivo es construir situaciones de aprendizaje donde, a partir de la oralidad, se realice la identificación de discursos diferentes a los de las tradiciones discursivas escolares para adherirlos al currículo. Esta metodología, si bien no es bilingüe por los fenómenos de desplazamiento lingüístico de los alumnos, es intercultural ya que retoma cuestiones iconográficas-simbólicas y discursivas de los pueblos de origen de los niños migrantes, así como el rescate de la memoria colectiva y ancestral a partir de diversos tópicos detonantes.
\end{abstract}

Palabras clave: intervención pedagógica, tradiciones discursivas, interculturalidad.

\begin{abstract}
Direction of Migrant Education of Jalisco is part of the Ministry of Public Education in Mexico. Its objective is to offer quality education to children from migrant rural worker's families in multiple states of this country. In Jalisco, educational attention presents a series of issues related to communicative competences. The project "Teaching Staff Professionalization on Migrant Education" is a plan of pedagogical intervention for written language implemented in four phases. The written language is fostered by an intercultural, de-colonial approach scheme in contexts where oral cultures prevail. The goal is to construct learning situations from orality to accomplish the identification of different discourses other than the academically traditional one in order to add them to the curricula. This method, while not bilingual because of language shift phenomena observed in the students, is indeed intercultural due to the fact that takes up discursive and iconographic-symbolic representations of the migrant children's native culture while using triggering topics to rescue the collective and ancestral memory.
\end{abstract}

Key words: pedagogical interventions, discursive traditions, interculturality. 


\section{INTRODUCCIÓN}

La Dirección de Educación Migrante es una institución que depende de la Secretaría de Educación Pública en México. El objetivo del programa es dar atención educativa de calidad a los niños, niñas y adolescentes, hijos de familias agrícolas migrantes internas en diversos estados de la República Mexicana, que buscan mejores oportunidades de empleo. En Jalisco, esta institución atiende, durante un ciclo escolar, a más de 2300 niños que cursan primaria y secundaria, según información arrojada por el Sistema Nacional de Control Escolar de Población Migrante (Sinacem), para el ciclo 2017-2018. Este organismo funciona como tal desde 2014, cuando entró en vigor el Programa de Inclusión y Equidad Educativa (PIEE); sin embargo, funcionó como Programa de la Niñez Migrante (Pronim) desde 1986.

La Dirección de Educación Migrante tiene en la actualidad 40 centros de trabajo en doce regiones de Jalisco: Cihuatlán, Tamazula de Gordiano, San Gabriel, Tolimán, Tuxcacuesco, Casimiro Castillo, Autlán de Navarro, El Grullo, Tequesquitlán, Tomatlán (Cruz de Loreto, José María Morelos, Pino Suárez y Campo Acosta y San Cristóbal de la Barranca; además de un plantel en la Zona Metropolitana de Guadalajara en la colonia Miramar, municipio de Zapopan.

Los migrantes con mayor presencia en Jalisco provienen de: Guerrero, Hidalgo, Morelos, Oaxaca y Chiapas; sin embargo, en las aulas de Educación Migrante cada vez hay más presencia de población de Veracruz, Sinaloa, Michoacán, Colima y producto de desplazamientos regionales y municipales del interior del estado hacia la Zona Metropolitana de Guadalajara, lo que da como resultado un crisol de diversidad lingüística y cultural al que se debe dar atención mediante procesos de inclusión educativa.

Uno de los principales problemas a los que se enfrentan los docentes de Educación Migrante es el rezago educativo; prueba de ello son los resultados que alumnos de este sistema obtienen en pruebas estandarizadas nacionales como PLANEA:

Los resultados de PLANEA 2015 demuestran que aún existen brechas importantes en cuanto al logro educativo de poblaciones en contexto de vulnerabilidad: 83 de cada 100 alumnos de escuelas 
indígenas y 70 de cada 100 estudiantes de escuelas comunitarias (PRONIM) están en el nivel más deficitario de logro o nivel de logro I (INEE, 2016b, p. 2).

Este tipo de resultados son preocupantes académicamente porque, en muchos de los casos, la prueba PLANEA no está contextualizada y el desempeño se califica, en general, por debajo de la media, por lo que, a largo plazo, detona en otras problemáticas como la deserción escolar o la ineficiencia terminal, lo que acaba por vulnerar más a estas poblaciones, ya que la baja escolaridad en muchas ocasiones no permite la movilidad social: "En materia educativa, por ejemplo, y de acuerdo con datos de la ENJO 2009, la escolaridad promedio de los jornaleros agrícolas migrantes para ese año era de 4.5 años, en comparación con la media nacional de 8.1 años" (Sedesol, 2011, s. p.).

A partir de 2015, la Dirección de Educación Migrante Jalisco comenzó un proyecto de intervención pedagógica, en formato de sesiones de profesionalización docente, con el objetivo de mejorar la calidad del servicio educativo y dotarlo de herramientas para que la atención a esta población fuera eficaz, pero, sobre todo, pertinente; es decir, en relación con la funcionalidad de los aprendizajes y la inclusión de otras visiones del mundo al currículo nacional. Para ello, se tomó como base el documento Directrices para mejorar la atención educativa de niñas, niños y adolescentes de familias de jornaleros agrícolas migrantes (2016), que propone un enfoque intercultural a partir de: "Alinear la oferta formativa al desarrollo de prácticas pedagógicas basadas en el enfoque intercultural y la atención a la diversidad, que conviertan en ventaja pedagógica la diversidad cultural y lingüística, el conocimiento sobre la agricultura, la migración y la experiencia de vida que tiene esta población escolar" (INEE, 2016b, p. 29).

La primera parte del proyecto, enfocada en competencias comunicativas, se dividió en cuatro fases de trabajo, de las cuales se explicarán las primeras dos etapas para este texto:

- Primera: sensibilización y diagnóstico participativo de los colectivos docentes. 
- Segunda: experimentación con grupos (alumnos de los albergues en Tamazula de Gordiano).

- Tercera: formación profesional.

- Cuarta: adecuación curricular y diseño de materiales.

Objetivo general: diseñar una estrategia de intervención educativa en favor de la calidad en los aprendizajes, en el área de competencias comunicativas de los alumnos pertenecientes a la Dirección de Educación Migrante Jalisco.

Objetivos específicos de las primeras dos etapas:

- Recuperar tradiciones discursivas de los pueblos de origen de los alumnos pertenecientes a Educación Migrante.

- Profesionalizar a los docentes de Educación Migrante en el uso de estrategias de aprendizaje con enfoque intercultural.

- Compilar las producciones escritas por los alumnos que pertenecen a Educación Migrante Jalisco para, posteriormente, diseñar material didáctico.

- Fomentar el uso de la oralidad como vehículo de conocimiento con autoridad dentro del aula.

\section{BILINGÜISMO Y TRADICIONES DISCURSIVAS}

En el estado de Jalisco existen 40 centros de trabajo pertenecientes a la Dirección de Educación Migrante. Según el SINACEM 2.0, para el periodo 2016-2017 había en matrícula 1317 alumnos en el nivel primaria. Los datos para alimentar el sistema se obtienen a través de las cédulas de inscripción que se llenan al inicio de ciclo, las cuales se componen de tres campos informativos: datos generales del alumno, datos de la escuela de adscripción e información sobre la situación migratoria de padres de familia y alumnos; en este último rubro, existe un apartado para registrar si el alumno es de origen indígena y si habla alguna de las 68 lenguas originarias mexicanas, además del español como lengua franca. A partir de esta sección, se puede percibir el fenómeno de desplazamiento lingüístico, es decir, el reemplazo de una lengua A por una B por motivos sociales, económicos y culturales, lo que ha ocurrido como consecuencia de la migración interna (Kabatek, 2005). 
Según Sinacem, las lenguas que más aparecen como lengua materna en la matrícula de Jalisco son: ñuu sáavi, náhuatl (variantes de Guerrero, Hidalgo y Morelos), purépecha, wixárika y español. Sin embargo, aunque en muchos casos los padres de familia y los alumnos se identifican como población indígena, no se asumen como hablantes de lenguas originarias no occidentales. Por ejemplo, la población en los albergues de Tamazula de Gordiano fue de alrededor de 110 niños para el ciclo 2018-2019; de este total poblacional, 13 niñas y 9 niños se identificaron como hablantes de una lengua materna distinta del espańol, en todos los casos, náhuatl de la variante guerrerense y de la huasteca hidalguense, por lo que se tiene que el $18 \%$ son hablantes de lenguas originarias.

Aunque lo ideal es que este $18 \%$ reciba educación en su lengua materna con un maestro bilingüe, las condiciones del servicio son adversas, ya que por la situación de movilidad, convive con otros alumnos con circunstancias socioculturales y lingüísticas distintas. Ello obliga a plantearse las siguientes preguntas: ¿Qué lengua se preferiría sobre cuál otra para la enseñanza sistémica? ¿Enseñar en español como lengua franca en contextos plurilingües vulnera los derechos lingüísticos de otras lenguas? ¿Cómo trabajar la inclusión en contextos plurilingües?

Al respecto, Ramos y Barriga (2019, p. 39) mencionan en su texto Prácticas lingüisticas y lenguas indigenas, entre historias, discursos, paradojas y testimonios:

La esencia pluricultural de México y la diversidad lingüística que de ella emana ha provocado a lo largo de la historia grandes paradojas plasmadas en políticas lingüísticas y educativas unilaterales -sean de incorporación, de integración o de autodeterminación-, contradictorias e inconsistentes todas ellas, que han conducido a un final, al parecer, irremisible: por un lado, el desplazamiento de las lenguas originarias por el espańol, lengua de prestigio y dominante; por el otro, la fractura y pérdida de identidad de sus hablantes.

El desplazamiento lingüístico, efecto de la discriminación, la violencia y la falta de oportunidades es una realidad en las aulas de Educación Migrante, sin embargo, la cultura, aunque encuentra 
un vehículo de comunicación eficaz en las lenguas originarias, al quedar en desuso, se sigue manifestando de otras maneras: a través de la gastronomía, la danza, las artes visuales, la música, todos ellos inmersos en discursos con la misma validez que la lengua misma y conformadores de identidad.

Uno de los principales cuestionamientos que emergen en estos contextos es cómo se puede atender la diversidad de manera eficaz y pertinente desde la intervención y la adecuación curricular. El enfoque bilingüe que es funcional en las escuelas adscritas al sistema de Educación Indígena en el área de migrantes tendría que considerarse de manera crítica, ya que no todos los alumnos son bilingües ni los niveles de bilingüismo son los mismos (Rotaetxe, 1996).

Hay que tomar en consideración que, incluso en las aulas de educación indígena, el bilingüismo es abordado desde visiones occidentales de la lengua. Por ejemplo, los libros de texto que se otorgan a los alumnos desde la Comisión Nacional de Libros de Texto Gratuitos (Conaliteg) son los mismos que se distribuyen en toda la República Mexicana, a excepción de un libro de lengua materna. Además, en muchos casos, el propio material, aunque en una lengua diferente del español, son traducciones. Tal es el caso de la lengua wixárika en el norte de Jalisco, para quien Ediciones Castillo publicó el libro Cuéntame, lecturas para todos los días en lengua wixárika, que constituye una compilación de cuentos y poemas hecha por Francisco Hinojosa, originalmente en español, por lo que la cosmovisión y discursos wixaaritari no se transmiten en este material. Lo mismo ocurre cuando la educación es bilingüe; no obstante, en el aula, la tipología textual se limita a la tradición occidental: fábulas, leyendas, poemas, cuentos tradicionales y, en muchas ocasiones, la lengua sirve sólo como soporte de discursos y no como creadora y transmisora de conocimiento.

Sin olvidar que es derecho de los niños recibir educación en su lengua materna, considerar dentro del currículo la enseñanza, valoración y uso de las tradiciones discursivas extraescolares, es decir, discursos estereotipados que se repiten en ciertas situaciones comunicativas en la vida cotidiana, es una de las propuestas que se pueden ofrecer en este contexto diverso: diferentes poblaciones, lenguas y percepciones sobre el servicio educativo. 
En este caso, el objetivo es reconocer, dialogar y posicionar otros discursos en el ámbito académico. Parte de la inspiración de esta propuesta es el uso del español por parte de movimientos sociopolíticos como el zapatismo: aunque de raíz indígena y en pleno uso de lenguas como el tzotzil o el tseltal, la Sexta Declaración de la Selva Lacandona está escrita en español, pero no es el español estándar o el discurso político con las formas retóricas del mismo, sino en su propia variante, que ellos retoman la tradición de la conseja oral y la llevan a la lengua franca:

Y entonces nuestra pequeña historia es que nos cansamos de la explotación que nos hacían los poderosos y pues nos organizamos para defendernos y para luchar por la justicia. Al principio no somos muchos, apenas unos cuantos andamos de un lado a otro, hablando y escuchando a otras personas como nosotros. Eso hicimos muchos años y lo hicimos en secreto, o sea, sin hacer bulla. O sea que juntamos nuestra fuerza en silencio (EZLN, 2005, s.p.).

En este sentido, se emplea el lenguaje cotidiano y conceptos bajo enfoques epistemológicos diferentes de los occidentales, además de que la fraseología parte de la variante dialectal del español en la montańa chiapaneca: se cumple el objetivo de la comunicación y el español se vuelve una herramienta a partir de la expresión en tradiciones discursivas propias.

Sin embargo, en el currículo a menudo no se toman en las potencialidades de la cultura escrita extraescolar. En lo que se refiere a la escritura, el plan de estudios encarna y reproduce las demandas sociales acerca de los textos y también su definición del procesamiento de textos como una actividad sujeta a determinadas normas de conducta... Los niños que, por cualquier motivo, no son capaces o no están dispuestos a adoptar la competencia lingüística que se privilegia en la escuela, serán asimismo renuentes a asimilar los géneros de escritura que se incluyen y promocionan en los planes de estudio oficiales. El modo como se produce y se interpreta un texto constituye esencialmente una función social (Pontecorvo, 2002, p. 134). 
Para este ejercicio de tradiciones discursivas también se toma en cuenta la posición predominante de la oralidad, no como un estadio que lleva a la lectoescritura, sino como un fenómeno en sí mismo con sus potencialidades (Ong, 2016). De esta forma todos los discursos que no forman parte del currículo oficial pueden aparecer como motivante de la comunicación dado que no podemos asumir que las demandas sociales de la lengua son las mismas en todo el territorio mexicano; por ejemplo, en una comunidad donde existe el consejo comunitario o las reuniones públicas, no se maneja el mismo discurso que en una colonia donde los vecinos no se organizan para cuestiones de convivencia.

\section{METODOLOGÍA}

Durante el periodo escolar 2015 2016, los asesores escolares y docentes frente a grupo fueron convocados por la Coordinación Académica del programa para realizar un diagnóstico participativo sobre el servicio educativo, que se conformó a partir de mesas de trabajo y con la participación del Instituto Nacional para la Evaluación Educativa (INEE) quienes, en su documento Directrices para mejorar la atención educativa... plantearon cuatro líneas para lograr ese objetivo. Para efectos de este trabajo, nos orientaremos por la segunda directriz "Asegurar la pertinencia de un modelo educativo intercultural, así como la disponibilidad, idoneidad y desarrollo profesional del personal con funciones de docencia" (INEE, 2016a, s.p.); ya que el objetivo general del proyecto de intervención educativa era mejorar la calidad del servicio, a partir de la pertinencia.

Desde el formato Consejo Técnico Regional se realizó un diagnóstico de la atención educativa hacia los grupos migrantes. En dichos espacios participaron cuatro directivos, 11 asesores escolares y 69 docentes, en total. Se trabajó a partir de entrevistas semiestructuradas que se focalizaban tanto en la percepción de la calidad educativa, cuanto en las problemáticas que ellos identificaban en el servicio. A continuación, describiremos el proceso: 
1. Las mesas de trabajo se dividieron en cuatro, una por cada directriz emitida por el INEE, en ellas había un secretario y un moderador.

2. La coordinación académica se enfocó en la mesa dos, conformada por dos directivos, cinco asesores escolares y 16 docentes.

3. Se aplicó una entrevista semiestructurada que se integró a partir de 45 reactivos de respuesta libre, divididos en tres apartados:

- Perfil profesional y prácticas docentes

- Perfil del alumno

- Atención administrativa

4. Los docentes respondieron de manera individual y, en un segundo momento, de forma colectiva. Los secretarios tomaron nota y desarrollaron una conclusión que fue leída al final de la reunión.

Las respuestas a los cuestionarios fueron procesadas a través de un análisis de frecuencia, a partir del cual se llegó a las siguientes conclusiones en cuanto al perfil del alumnado, cuestiones que los docentes seńalaron como problemas dentro del aula:

- Alumnos cuya lengua materna no es el español, o plurilingüismo en una misma aula, es decir, hablantes de lenguas diferentes en un mismo salón o convivencia de diversas variantes dialectales de las lenguas.

- Alumnos que no leen ni escriben en grados donde es una competencia comunicativa necesaria.

- La disciplina de los alumnos en el aula.

A partir de la frecuencia de respuestas, es notable que la mayoría de las áreas consideradas "problemáticas" se relacionan con las competencias comunicativas. A continuación, se muestra la descripción de la primera etapa del proyecto de intervención pedagógica, que se inclina hacia las competencias comunicativas, y cuya ejecución y muestreo se llevó a cabo en la comunidad de Tamazula de Gordiano: 


\section{Primera etapa: Sensibilización de los colectivos docentes}

La primera etapa del proyecto de profesionalización consistió en un ejercicio de sensibilización de los colectivos docentes. Las jornadas de trabajo se llevaron a cabo en dos sesiones: durante marzo y noviembre del 2015, en las que participaron los asesores escolares de las 11 regiones que se atienden en Jalisco (imagen 1). Con la colaboración de la UNESCO, en ellas se trabajaron temas de interculturalidad y de inclusión lingüística. Las acciones más relevantes de las jornadas fueron:

- Exposición sobre el estado de las lenguas indígenas en México.

- Concepto de lengua, lenguaje y dialecto, así como de las variantes dialectales tanto de lenguas originarias como del español.

- Análisis del nivel de lengua de los libros de texto.

- Métodos básicos de observación de diversidad lingüística en el aula.

- IMAGEN 1. Reunión de diagnóstico, Vallarta 2016

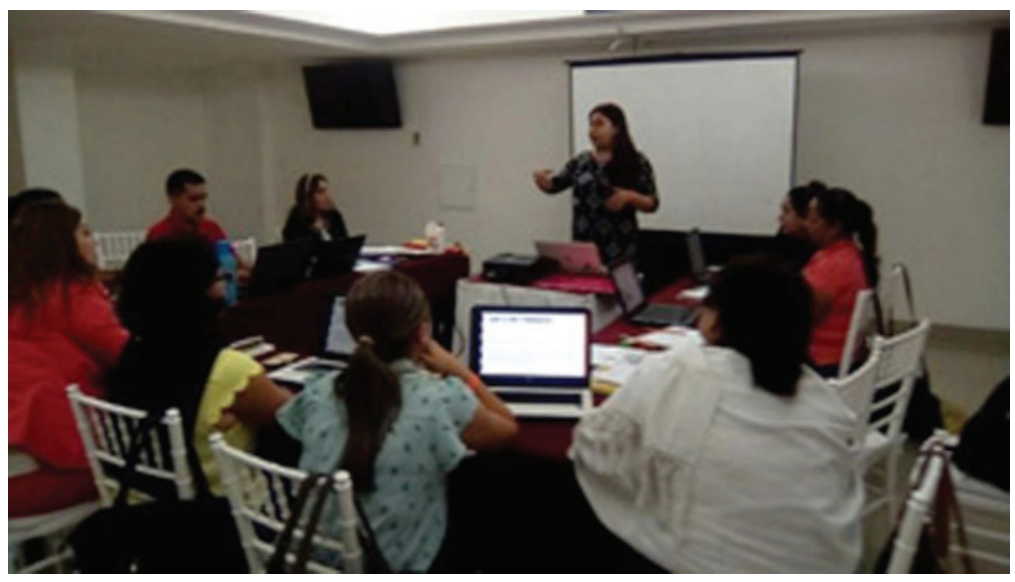

Fuente: imagen de archivo de campo.

\section{Segunda etapa: Experimentación con grupos}

La segunda etapa del proyecto consistió en un ejercicio de observación desde el punto de vista sociolingüístico. Se identificaron de ma- 
nera superficial los niveles de lengua y variantes dialectales a partir del Atlas Lingüístico de México y el registro de los lugares de origen de los alumnos; además, se recuperaron textos donde quedaban de manifiesto algunas de las tradiciones discursivas de los lugares de origen de los alumnos, que era el objetivo primordial de esta etapa, con el fin de construir una metodología de trabajo de didáctica de la lengua oral y escrita para niñas, niños y adolescentes hijos de jornaleros agrícolas. Durante esta fase se trabajó en dos albergues del municipio jalisciense de Tamazula de Gordiano: Arroyo Hondo y Ex Hacienda.

El uso del español como lengua franca podría parecer una medida colonizante; sin embargo, a diferencia de la atención a grupos indígenas, en un aula de migrantes la diversidad lingüística es tanta, que sería poco menos que imposible atender la realidad de cada niño. Además, se toma en consideración que mucha de la población infantil migrante está en proceso de desplazamiento lingüístico, por lo que muchos niños, aunque tengan padres de origen indígena, hablan el espańol como lengua materna. Es por ello que la propuesta del proyecto, además de fomentar el uso y reconocimiento de las lenguas originarias como riqueza lingüística, propone la documentación y uso en las aulas de las tradiciones discursivas diferentes de las del español escolar.

Los talleres partieron de dos hipótesis: la primera es que, si bien la normatividad del español es importante, para las poblaciones donde predominan las culturas orales la didáctica debe ser más flexible y permisiva, con respecto a la norma culta. Al respecto Ávila (1986, p. 16) afirma:

puede ser descrito, sin eufemismos, como lo hacen Emilia [Ferreiro] y Ana [Teberosky] "es regularmente el modo de habla de la clase dominante del centro político de país”. Frente al modelo, los usos divergentes son considerados incorrectos -barbarismos, solecismos, vulgarismos y otros ismos- y se rechazan sintomáticamente en la escuela y en los grupos sociales privilegiados.

El respeto y la inclusión de las variantes dialectales del español y su presencia en la lengua escrita son fundamentales. Los alumnos de las aulas migrantes pertenecen a la tradición oral, pero al escolarizar- 
se deben aprender la lengua escrita; no obstante, si el profesor incide demasiado en el uso de la norma de la lengua escrita, el alumno perderá el interés y la lengua escrita le parecerá poco funcional, ya que lo que habla y lo que se escribe es distinto:

Si un niño es campesino, probablemente aprendió a decir haiga, váyamos, juites, estrumento, inorante, oyir, y no las formas estándar. Para él esa pronunciación es absolutamente legítima... Imaginemos que a nosotros nos piden que escribamos no como mexicanos sino como asturianos: tendríamos que aprender el dialecto de Asturias mediante reglas, y seguramente preferiríamos no escribir (Ávila, 1986, p. 17).

La segunda hipótesis se relaciona con las tradiciones discursivas. Se entiende por tradición discursiva las "formas tradicionales de decir las cosas, formas que pueden ir desde una fórmula simple hasta un género o una forma literaria compleja" (Kabatek, 2005, p. 156). Al respecto, uno de los errores frecuentes que se cometen en las escuelas durante la enseñanza de lenguas es construir didácticas para la notación de una lengua o privilegiar unas tradiciones discursivas sobre otras. Por ejemplo, en una escuela con niños wixaáritari se puede enseñar la lectura y escritura de la lengua wixárika: los grafemas, la construcción de oraciones, los blancos gráficos, pero el docente se focaliza en cómo "escribir" desde el punto de vista biomecánico y cognitivo del código, y no desde las prácticas sociales. Además, enseñará durante la clase el discurso "fábula" en lugar de privilegiar la enseńanza sistematizada de su propia tradición discursiva, como el formato uxátsika, que es un tipo de discurso narrativo con sus propias intenciones comunicativas, más allá de las ficcionales o estéticas de un cuento occidental. A la lengua escrita se le exime de su papel sociocultural:

existe una gran diferencia entre hacer la de una lengua, en este sentido técnico, y una lengua, entendiendo por ello que las escrituras existen como fenómenos dentro de una sociedad, inmersos en la historia, y que no están fundados sólo en consideraciones técnicas de ese tipo (Benveniste, 2002, p. 15). 
A partir de las hipótesis anteriores comenzó el trabajo de campo. Los objetivos del proyecto que se trabajaron en esta fase fueron:

- Recuperar tradiciones discursivas de los pueblos de origen de los alumnos pertenecientes a Educación Migrante.

- Compilar las producciones escritas por los alumnos pertenecientes a Educación Migrante Jalisco.

La ejecución del taller estuvo a cargo de la Coordinación Académica de la Dirección de Educación Migrante. Se gestionaron recursos estatales para la investigación de prácticas discursivas a través del Fondo Especial para el Desarrollo Cultural Infantil "Alas y Raíces Jalisco" durante el ciclo agrícola noviembre-junio de 2016 a 2018, en el municipio de Tamazula de Gordiano, albergue cańero "Soyatlán" y "Ex Hacienda"; así como en San Gabriel, Albergue de Bioparques S. A. de C. V.

Los talleres se ofertaron los sábados como espacios para la escritura creativa y la promoción de las artes, y duran tres horas. Durante los dos ciclos migratorios se atendió a un promedio de 120 niños de entre 6 y 14 años, todos hijos de jornaleros agrícolas migrantes.

Durante el trabajo se identificaron dos lenguas originarias habladas por los participantes: náhuatl de la variante de Guerrero y de la Huasteca hidalguense, así como tu'un savi, también de la variante de Guerrero. Sin embargo, es importante seńalar que, de los aproximadamente 120 nińos atendidos, sólo seis de ellos hablaban alguna lengua originaria, el resto hablaba espańol como lengua materna.

Los ciclos de talleres se realizaron de 2016 a 2017 y de 2017 a 2018; en ambos casos, se trabajó bajo la modalidad de taller a partir de técnicas de animación sociocultural. Cada sesión comenzaba con la narración oral de un cuento o anécdota que detonaba en tres variantes textuales: narración, explicación u opinión. Del primer ciclo, 2016-2017, se obtuvo:

\section{Tópicos recurrentes}

A partir de diagnósticos participativos, los alumnos pudieron aportar ideas para la construcción de los talleres. Los talleristas propo- 
nían temáticas y los alumnos realizaban elecciones para las sesiones subsecuentes. Los tópicos más recurrentes fueron: seres sobrenaturales (brujas, chaneques, nahuales, diablos), conocimiento sobre la agricultura, historias sobre viajes o anécdotas, fantasmas aparecidos y supersticiones, memorias familiares, narraciones o información sobre animales, narraciones de sueños, deseos sobre el futuro o adivinación y tradiciones de los lugares de origen.

\section{Recuperación de conocimientos y saberes de los alumnos a partir de un corpus textual}

La finalidad de los talleres no era la de enseñar gramática textual, por lo que empleamos instrumentos tipo ensayo, en los que los alumnos mostraban, a partir de sus producciones escritas, las nociones que tiene sobre el texto; es decir, los talleres no tenían como objetivo enseñar textualidad ni normas, sino permitir a los alumnos expresar sus ideas y realizar la construcción del texto a partir de sus conocimientos previos, tanto en el contenido como en la forma. Por ejemplo, durante una de las sesiones de trabajo se comenzó contando historias de espantos y aparecidos, tema que era de la preferencia de los niños. Los niños comenzaron a contar historias que les habían sucedido a ellos o a sus familiares. Posteriormente, la instrucción era que entre todos se diseñaría un libro de estas historias con el fin de preservar la memoria. No hubo instrucciones de cómo comenzar el texto, ni de la extensión o las partes del mismo.

El siguiente ejemplo es muy interesante: la alumna que realizó la imagen 2 no contó una historia, es decir, no utilizó el género narrativo, sino que escribió una creencia arraigada en su pueblo, utilizó el elemento retórico de inicio "Dicen que..." y, de este modo, nos permite apreciar cuáles son los géneros discursivos que ella identifica de su realidad familiar.

Uno de los principales objetivos era obtener un corpus de textos que nos permitiera comprender la lógica de las producciones, así como reconocer a priori las formas del discurso que los alumnos utilizaban normalmente en sus intervenciones. $\mathrm{Al}$ principio, los alumnos utilizaban fórmulas de la tradición discursiva escolar como "Ha 


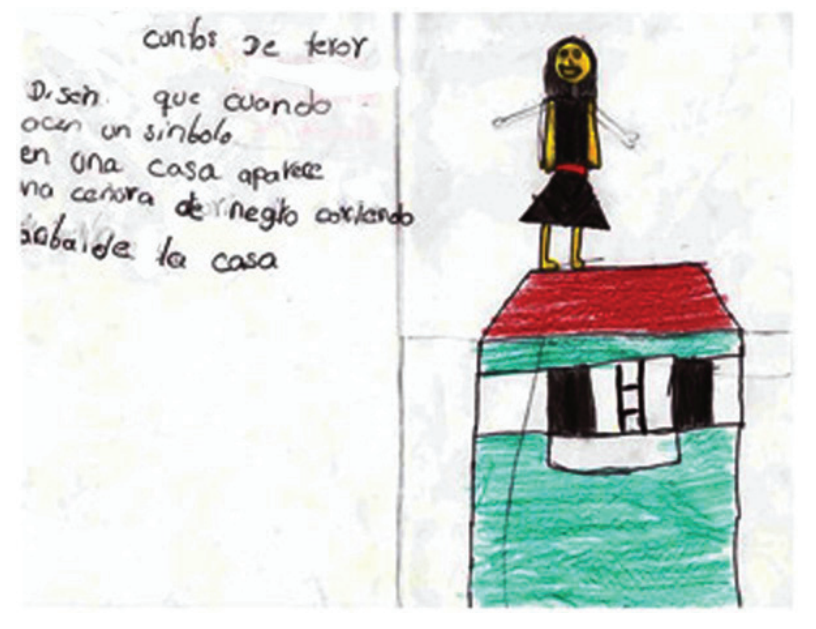

Fuente: imagen de archivo de campo.

bía una vez" o "Érase una vez", porque en las aulas es una práctica común, incluso forzaban finales parecidos a los de los cuentos tradicionales europeos con fórmulas como "Fin". A continuación, analizaremos un ejemplo: durante un mes, en el ciclo 2017-2018 en Tamazula de Gordiano, se trabajó con flora y fauna mexicana con el fin de rescatar los conocimientos de los alumnos sobre estos temas. A partir de sus saberes, se trabajó en álbumes de insectos, aves, plantas, frutos, entre otros. En una de las sesiones se les pidió que realizaran descripciones de animales. En el texto siguiente (imagen 3), una niña de origen guerrerense escribió un cuento sobre animales empleando fauna mexicana, un lobo y un conejo, pero con una estructura de cuento tradicional: es notable el empleo de la fórmula retórica "Érase una vez" frase que la alumna escribe separando el verbo, para terminar el cuento con un "Fin".

La pretensión inicial era crear ambientes horizontales de trabajo para que las producciones fueran lo más espontáneas posible, como ya se explicó anteriormente. Para los talleres se procuró elegir textos detonantes que tuvieran estructuras diversas. Entre los materiales más usuales estuvieron los libros de literatura editados por el Consejo Nacional de Fomento Educativo (Conafe), así como otros textos relacionados con narrativas no occidentales como la colección de video-cortometrajes titulada 68 voces, 68 corazones proyecto de 
Gabriela Badillo a partir de una beca del Fondo Nacional para la Cultura y las Artes (Fonca); o los cortometrajes de Consejo Nacional para la Cultura y las Artes (Conaculta) titulados Ventana a mi comunidad.

- IMAGEN 3. Producción escrita. Tamazula de Gordian. Periodo 2016-2017

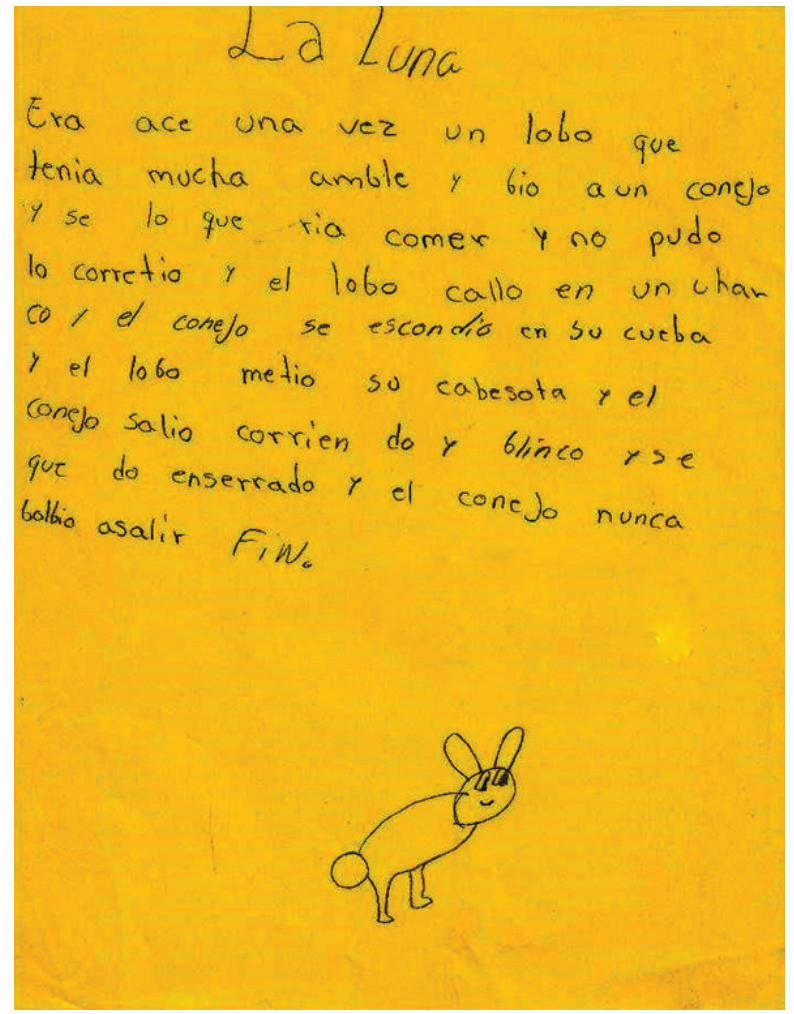

Fuente: imagen de archivo de campo.

Si bien la muestra puede estar sesgada por los detonantes que se usaron, es importante que antes de cualquier producción exista un diálogo entre los talleristas y los alumnos, es decir, que los alumnos se sientan partícipes de lo que está sucediendo en el aula y que no sean corregidos de manera reiterada. En este caso el diálogo siempre giraba en torno al tema de la sesión.

Durante el segundo ciclo, 2017-2018, y a partir de los resultados del primer taller, se trabajó la escritura focalizada en tópicos recurrentes. Lo que se obtuvo se describe a continuación. 


\section{Corpus textual diverso}

A partir de ejercicios de escritura creativa y escritura derivada se obtuvo un corpus de textos que pertenecen principalmente a tres tipologías textuales: la narración, la exposición y la descripción; entre los textos hubo quienes seguían las reglas de la textualidad escolar, pero otros mostraban formas discursivas de sus pueblos de origen, normalmente adaptadas de la oralidad a la escritura en el género de "creencias", que utiliza el elemento discursivo "Dicen que...", que se muestra en la imagen 4. En un primer momento, la alumna comienza a hablar de un lugar mítico en su pueblo de origen, un pozo, del que salen seres mágicos denominados chaneques y continúa no como texto narrativo, sino como creencia de sucesos mágicos. Además, conlleva los elementos "dicen que" y la causalidad, si sucede la condición A, en este caso la lluvia; entonces, lleva a una situación B: la aparición de los chaneques, que constituye, en su totalidad, una superstición ya conformada en el imaginario colectivo.

- IMAGEN 4. Producción escrita. Tamazula de Gordiano. Ciclo 2017-2018

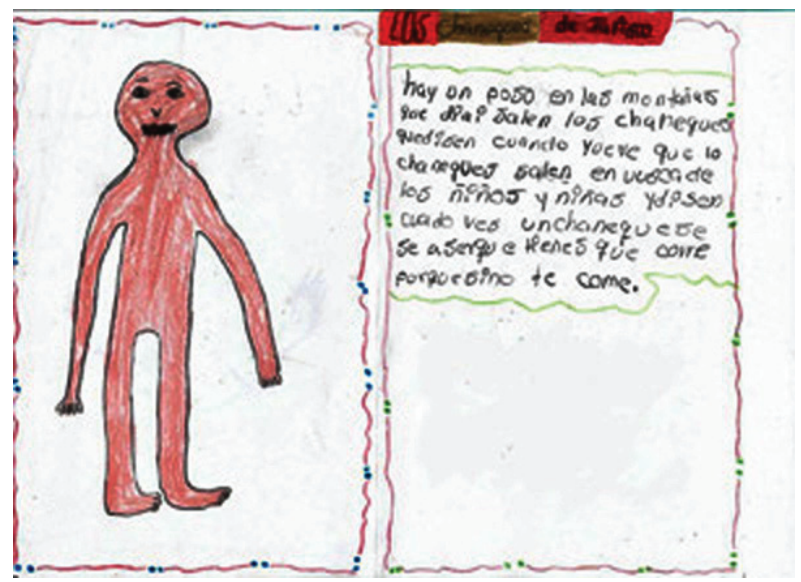

Fuente: imagen de archivo de campo.

Del corpus obtenido durante este ciclo se editó el libro Tejiendo la Memoria (Figueroa y López, 2019) que compila las producciones escritas de los niños, así como sus ilustraciones, con el fin de que sirva como insumo de lectura durante las clases. Los textos no fueron editados más allá de correcciones de estilo relacionadas con 
la legibilidad de los textos, se respetaron las variantes dialectales, así como la organización original de los textos. El libro se compone de los textos originales escaneados, el texto transcrito y las ilustraciones de apoyo.

\section{Manifestaciones iconográficas no estandarizadas}

Parte medular del proyecto era la identificación de tradiciones discursivas visuales, además de las orales y escritas. De manera regular, los niños se encuentran en un ambiente donde las caricaturas y los medios audiovisuales que les rodean son producidos mayoritariamente por países de cultura occidental, en nuestro caso por Estados Unidos. Creemos que también las representaciones visuales se han ido colonizando, por lo que la forma en la cual los niños ven el mundo está filtrada por estas representaciones. Por ejemplo, la representación de un árbol. Si le pedimos a cualquier persona que no sea artista visual que dibuje un árbol, hará una representación general del mismo: un tronco café con una copa verde y esponjada, que son las representaciones generales de árboles que aparecen en las caricaturas y en algunos libros infantiles. Sin embargo, dada la riqueza de conocimiento que tienen las niñas y niños hijos de jornaleros agrícolas migrantes sobre plantas, durante los talleres también se fomentó la representación de las mismas, por ejemplo, la ilustración de un huerto (imagen 5).

Se obtuvieron representaciones visuales a partir de diversas técnicas visuales: bordados, tejidos, papel picado, papel maché, barro, acuarelas, colores pastel, iluminación, entre otros. Por ejemplo, la imagen 6 fue diseñada por uno de los participantes de los talleres. El consenso en esta actividad fue realizar un manual de siembra para otros niños que no supieran cómo realizar esta actividad. Los participantes del taller realizaron sus instructivos y los ilustraron a partir de su propia experiencia en el campo y no como una copia de otras ilustraciones al respecto. 
IMAGEN 5. Producción textual y visual. Flora ubicada en los huertos. Tamazula de Gordiano. Ciclo 2017-2018

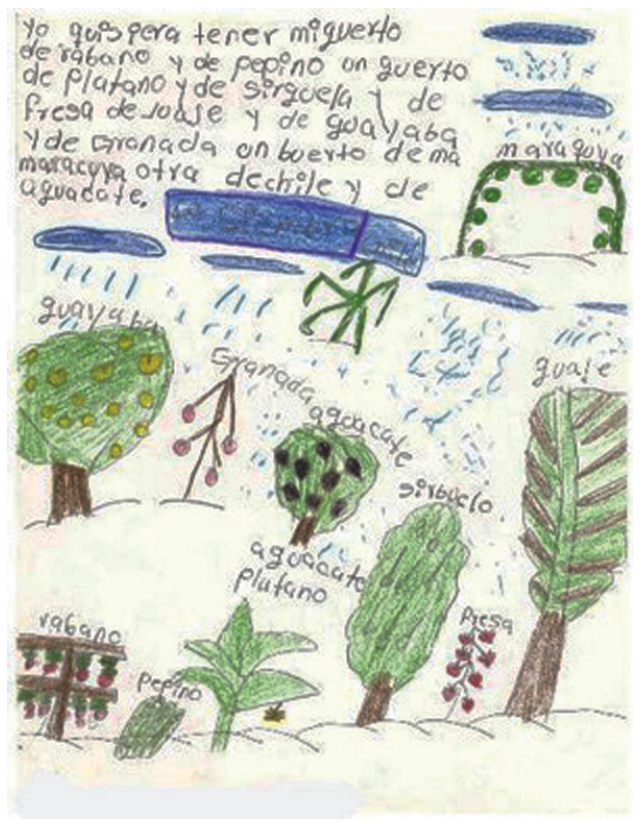

Fuente: imagen de archivo de campo.

- IMAGEN 6. Dibujo sobre la siembra, diseñado por alumno participante en los talleres Tejiendo la memoria en la comunidad de Soyatlán, municipio de Tamazula de Gordiano en Jalisco. Ciclo 2017-201 8

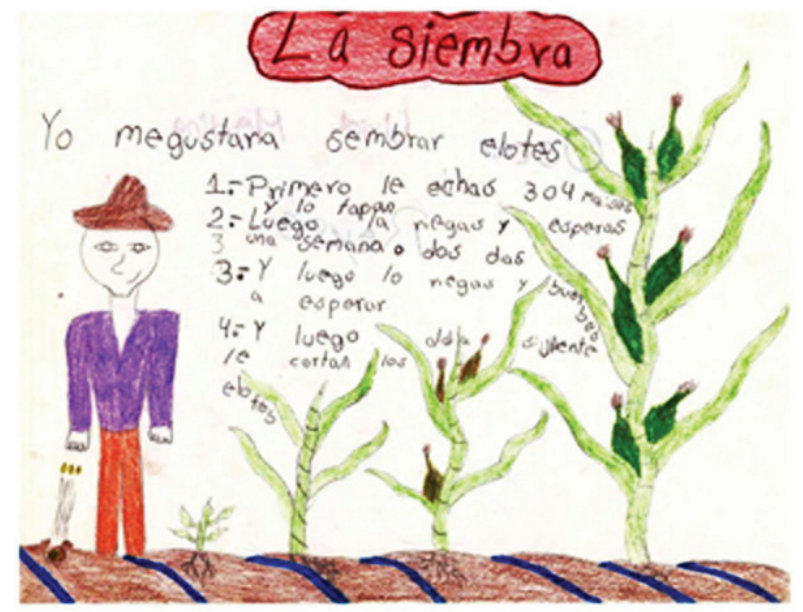

Fuente: imagen de archivo de campo. 


\section{CONCLUSIONES}

A manera de conclusión, es necesario mencionar que, a partir de los corpus obtenidos durante los talleres, aún faltan diversos análisis para realizar una intervención pedagógica pertinente y significativa en el área de lengua; es decir, que los materiales que ya se obtuvieron pueden servir como modelo para otros nińos del nivel o para la composición de materiales con un nivel de lengua más acercado a las variantes dialectales de los alumnos.

La intención en todo momento es reconocer las culturas de origen de los niños para afianzar la identidad, ya que muchos de ellos permanecerán durante años sin visitar sus comunidades. También es necesario considerar que están en constante cambio y que el modelo educativo que se les ofrezca debe ser intercultural y multidialectal, de manera que la forma en la que nombran la realidad o los objetos, aparezca también en las secuencias didácticas, además de que conozcan las formas de ser de otras comunidades nacionales $u$ otros pueblos originarios en el mundo, y de que no se debe promover la prevalencia de una cosmovisión del mundo sobre otra. Para los niños la escritura debe significar un método de liberación, de expresión y de acción social, no una imposición docente que terminará por no parecerles útil, de acuerdo con la visión de educación popular de Paulo Freire.

Si bien mucha de la población infantil no alcanzará grados superiores de estudio, se espera que su paso por la Educación Básica en las aulas de la Dirección de Educación Migrante sea fructífera, respetuosa con sus prácticas socioculturales y pertinente, para evitar la deserción escolar, el rezago educativo y la ineficiencia terminal.

Los niños migrantes, así como sus visiones del mundo transmitidas a través de la oralidad, la escritura y cualquier otra manifestación visual deben ser incluidas en las aulas, en la escolarización formal y en los constructos que se van diseñando en el aula, ésta es una manera de lograr la inclusión y la equidad de manera tangible más allá de los discursos.

La construcción curricular, además, debe incluir no sólo las tradiciones discursivas de los pueblos de origen de los niños: las historias de animales, los mitos originarios, los almanaques de viajes, las 
lecciones de los abuelos, los refranes y consejas, entre otros, sino que deben mostrar que la realidad del mundo occidental generalizado no es la realidad de todas las infancias de América Latina y, en particular, de México. Contenidos temáticos como las estaciones del año ya conllevan una carga colonizante, cuando los nińos asumen que en invierno debe nevar, aun cuando nuestros climas distan mucho de estos paisajes. Que los materiales didácticos no sean hechos para nińos de determinado nivel socioeconómico, donde las casas aparecen con sus techos de dos aguas y con habitaciones para cada habitante, realidades que difieren mucho de las que viven los nińos migrantes, por ejemplo, con sus casas de un solo cuarto con corrales grandes.

Es necesario que la educación sea por y para los nińos, que exista un currículo donde quepan todas las visiones del mundo, un currículo verdaderamente intercultural que permita el diálogo, la cultura para la paz y la inclusión de todas las voces que conforman las infancias, en este caso, las mexicanas.

\section{REFERENCIAS BIBLIOGRÁFICAS}

Ávila, R. (1986). Sólo para tus oídos. En L. F. Lara y F. Garrido (coords.), Escritura y alfabetización (pp. 11-35). México: Editores del Ermitaño.

Benveniste, C. B. (2002). La escritura, irreductible a un código. En E. Ferreiro (comp.), Relaciones de (in) dependencia entre oralidad y escritura (pp. 15-30). España: Gedisa.

EZLN (2005). Sexta declaración de la Selva Lacandona. México: Enlace Zapatista. Recuperado de http://enlacezapatista.ezln.org.mx/sdsl-es/

Figueroa, M. T., y López, F. (coords.) (2019). Tejiendo la Memoria. México: Colectivo Milotli.

INEE (2016a). Directrices para mejorar la atención educativa de niñas, niños y adolescentes de familias de jornaleros agricolas migrantes. México: INEE. Recuperado de https://www.inee.edu.mx/images/stories/2014/ Normateca/Directriz_migrantes1.pdf

INEE (2016b). Directrices para mejorar la atención educativa de los niños, niñas y adolescentes de familias de jornaleros agricolas migrantes. Propuesta aprobada por la Junta de Gobierno Acuerdo SEJG/19-16/03. México: INEE. Recuperado de https://www.inee.edu.mx/wp-content/ uploads/2019/04/Directrices-Migrantes.pdf 
Kabatek, J. (2005). Tradiciones discursivas y cambio lingüístico. Lexis, XXIX(2), 151-177. Recuperado de http://revistas.pucp.edu.pe/index. php/lexis/article/viewFile/8387/8703

Ong, J. W. (2016). Oralidad y escritura: tecnologías de la palabra. México: Fondo de Cultura Económica.

Pontecorvo, C. (2002). Las prácticas de alfabetización escolar. En E. Ferreiro (comp.), Relaciones de (in) dependencia entre oralidad y escritura (pp. 133-149). España: Gedisa.

Ramos, G. C., y Barriga, R. (2019). De Babel a Pentecostés. Políticas lingüísticas y lenguas indígenas, entre historias, discursos, paradojas y testimonios. Lingüistica Mexicana. Nueva Época, 1(2), 186-190. Recuperado de http://linguisticamexicana-amla.colmex.mx/index.php/ Linguistica_mexicana/article/view/331

Rotaetxe, A. K. (1996). Lenguaje y sociedad: sociolingüística. En V. C. Martín (ed.), Elementos de lingüistica (pp. 307-339). Barcelona: Octaedro.

Secretaría de Desarrollo Social (2011). Pobreza, migración y capacidades básicas en la población jornalera agrícola en México. Resultados de la encuesta nacional de jornaleros agricolas 2009. México: Sedesol. Recuperado de http://www. cipet.gob.mx/jornaleros/ 